\title{
Planète Terre : des dangers annoncés
}

Hervé Le Treut

\section{(2) OpenEdition}

Journals

Édition électronique

URL : https://journals.openedition.org/histoire-cnrs/9052

DOI : 10.4000/histoire-cnrs.9052

ISSN : 1955-2408

Éditeur

CNRS Éditions

Édition imprimée

Date de publication : 5 octobre 2009

ISSN : 1298-9800

Référence électronique

Hervé Le Treut, «Planète Terre : des dangers annoncés », La revue pour I'histoire du CNRS [En ligne], 24 I 2009, mis en ligne le 05 octobre 2009, consulté le 20 mai 2021. URL : http://journals.openedition.org/ histoire-cnrs/9052 ; DOI : https://doi.org/10.4000/histoire-cnrs.9052

Ce document a été généré automatiquement le 20 mai 2021.

Comité pour l'histoire du CNRS 


\title{
Planète Terre : des dangers annoncés
}

\author{
Hervé Le Treut
}

1 Il est difficile de dater précisément le moment où la communauté scientifique a réellement commencé à diffuser un message d'alerte sur les risques associés à la croissance atmosphérique des gaz à effet de serre, mais une première étape décisive a certainement été le «rapport Charney» préparé pour l'Académie des sciences américaine, en 1979, et qui reste souvent cité. Sur la base de deux modèles et d'une estimation approximative de leur incertitude, ce rapport établissait une fourchette de réchauffement global de $1,5^{\circ} \mathrm{C}$ à $4,5^{\circ} \mathrm{C}$ pour un doublement du $\mathrm{CO}_{2}$ atmosphérique. Les modèles utilisés à cette époque étaient des modèles essentiellement atmosphériques, à la résolution grossière, comprenant des représentations encore simplistes des conditions de surface continentales ou océaniques.

2 Le Groupe intergouvernemental d'experts sur l'évolution du climat (GIEC) a été fondé en 1988 sous la double tutelle de l'Organisation météorologique mondiale (OMM) et du programme Environnement des Nations Unies, avec un objectif limité et précis, qui est souvent mal compris : permettre une synthèse, puis une transmission aux décideurs, des connaissances de la communauté scientifique. Le GIEC ne réalise aucune recherche, même si l'impact de ses rapports incite une part croissante de la communauté scientifique à se tourner vers la thématique du changement climatique. La recherche est en fait organisée au niveau international par des programmes dédiés tels que le programme mondial de recherche climatique (créé en 1990 sous l'égide de l'OMM, sur la base d'une action antérieure limitée à l'atmosphère, et dont le premier directeur a été le professeur Pierre Morel), ou le programme international Géosphère-Biosphère.

3 L'existence d'une communauté scientifique autonome et organisée a certainement constitué l'une des conditions qui ont rendu possible le travail du GIEC. Le premier rapport du GIEC est paru en 1990. Son diagnostic, s'appuyant sur les modèles de l'époque, reste remarquable encore aujourd'hui par la pertinence qu'il a conservée.

Il montrait qu'une augmentation continue des émissions de gaz à effet de serre, au-delà d'un réchauffement global, se caractérisait par une réponse très hétérogène 
géographiquement: augmentation de température plus intense dans les régions polaires arctiques, ou sur les continents; modifications du cycle hydrologique de la planète, avec un changement des régimes de précipitations conduisant en moyenne à une accentuation des contrastes actuels, les régions humides devenant plus humides et les régions sèches, plus sèches.

Depuis cette date, les modèles utilisés pour les scénarios de changement climatique futur ont fortement évolué, l'étape la plus importante ayant probablement été le couplage avec les modèles d'océan. En 1990, un seul des modèles présentés dans le rapport du GIEC intégrait une représentation explicite de la circulation océanique. Dans le troisième rapport, paru en 2001, il s'agissait là d'une norme respectée par tous, au prix d'un effort important poursuivi par toutes les équipes de recherche pendant plus d'une décennie. La complexification des modèles a touché bien d'autres domaines: physique des nuages, du sol continental et de son hydrologie, représentation de la glace de mer, introduction d'une biochimie destinée à comprendre les interactions entre cycle du carbone et climat, ou encore, plus récemment, prise en compte d'une chimie atmosphérique du méthane ou de l'ozone.

Un fait remarquable est que ces modèles plus complexes, qui ont été permis par l'augmentation continue des capacités de calcul durant les dernières décennies, ont pour l'essentiel confirmé la prévision des modèles plus anciens et plus simples, montrant que les mécanismes fondamentaux du climat sont des mécanismes robustes, et réfutant ainsi une part des arguments de simplisme opposés à la première génération de modèles. Parallèlement, la communauté scientifique s'est organisée autour de programmes destinés à évaluer quantitativement les résultats des modèles. Ces « MIP » (Model Intercomparison Programme: AMIP pour l'atmosphère, CMIP pour les modèles couplés, PMIP pour les paléoclimats, C4MIP pour le cycle du carbone, ou encore désormais CFMIP pour le rôle des nuages, pour ne citer que quelques exemples) ont certainement permis d'élever significativement la qualité générale des modèles, et ont défini peu à peu une stratégie de comparaison des résultats de modèles aux données observées. Ces exercices ont été fortement relayés par les rapports du GIEC, mais développés indépendamment du GIEC.

7 Cet effort important d'organisation et de structuration de la communauté internationale rend d'autant plus remarquable et préoccupant de constater, au-delà du consensus signalé plus haut, la persistance d'une dispersion importante des résultats des modèles en ce qui concerne à la fois l'amplitude précise des changements futurs et leur distribution régionale. La diversité des résultats n'a sur ces points pas été fortement diminuée. Il s'agit là d'une limitation considérable pour des études appliquées visant par exemple à utiliser ces modèles pour déterminer des objectifs précis de limitation des gaz à effet de serre, ou des mesures préventives d'adaptation aux changements à venir. Cette situation montre que le développement des modèles constitue toujours une priorité, malgré une histoire déjà longue de plusieurs décennies. Ce développement peut s'appuyer à la fois sur la perspective d'une augmentation toujours importante de la puissance des calculateurs, mais aussi sur l'apparition de données nouvelles. Pour ne donner qu'un exemple, le «train Aqua » dont les données permettent désormais de valider de manière beaucoup plus approfondie la sensibilité des modèles à des processus clefs telles que la physique des nuages, rassemble des satellites qui ont tous été lancés dans les dernières années. 
8 Mât instrumenté de la station du Puy de Dôme : taux de photolyse (décomposition chimique par la lumière) des molécules de $\mathrm{NO}_{2}$, humidité relative $(\mathrm{RH})$ et température ; prise d'air pour les espèces gazeuses. Les nuages modifient les propriétés oxydantes et radiatives de l'atmosphère en altérant les gaz à effet de serre et en lessivant/ altérant les aérosols. Pour connaître l'impact des différents types de nuages sur le climat, il faut notamment évaluer leur rôle sur la redistribution et les transformations chimiques des composés gazeux et particulaires à différentes altitudes. (C) CNRS Photothèque/ Laurence Médard

\section{RÉSUMÉS}

Le changement climatique global est aujourd'hui une réalité, et des indications toujours plus nombreuses montrent qu'il est largement dû aux activités humaines. C'est parce que la communauté scientifique s'est organisée, depuis plusieurs décennies, pour développer un diagnostic étayé, et le relayer auprès du grand public ou des décideurs, qu'une véritable prise de conscience des enjeux de cette évolution a pu se développer.

\section{AUTEUR}

\section{HERVÉ LE TREUT}

Spécialiste de la modélisation numérique du système climatique et de la compréhension des perturbations radiatives du climat, Hervé Le Treut dirige l'institut Pierre-Simon-Laplace. 